\title{
Effects of dark matter annihilation on the first stars
}

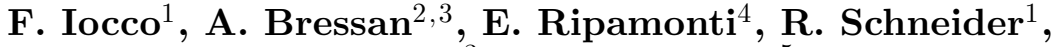 \\ A. Ferrara $^{3}$, and P. Marigo ${ }^{5}$ \\ ${ }^{1}$ INAF-Oss.Astr.di Arcetri; Largo E. Fermi 5, 50125 Firenze, Italy \\ ${ }^{2}$ INAF/Osservatorio Astronomico di Padova; Vicolo dell'Osservatorio 5, Padova, Italy \\ ${ }^{3}$ SISSA; Via Beirut 4, Trieste, Italy \\ ${ }^{4}$ Università degli Studi dell'Insubria, Dip. di Scienze Chimiche, Fisiche e Naturali; \\ Via Valleggio 12, Como, Italy \\ ${ }^{5}$ Università degli Studi di Padova, Dip. di Astronomia; Vicolo dell'Osservatorio 3, Padova, Italy

\begin{abstract}
We study the evolution of the first stars in the universe (Population III) from the early pre-Main Sequence (MS) until the end of helium burning in the presence of WIMP dark matter annihilation inside the stellar structure. The two different mechanisms that can provide this energy source are the contemporary contraction of baryons and dark matter, and the capture of WIMPs by scattering off the gas with subsequent accumulation inside the star. We find that the first mechanism can generate an equilibrium phase, previously known as a dark star, which is transient and present in the very early stages of pre-MS evolution. The mechanism of scattering and capture acts later, and can support the star virtually forever, depending on environmental characteristics of the dark matter halo and on the specific WIMP model.
\end{abstract}

Keywords. cosmology: early universe, cosmology: dark matter, stars: formation

\section{Introduction}

Within the scenario of a $\Lambda \mathrm{CDM}$ cosmology, it has been recently recognized that if the dark matter (DM) dominant component is a weakly interacting particle (WIMP), its annihilation could play a relevant role on the formation and evolution of the first baryonic objects in our universe. Spolyar, Freese, and Gondolo (2008) noticed that during the proto-stellar phase, the cooling of the baryonic gas could be overcome by the energy deposition following the annihilation of DM concentrated in the star formation site. This is a consequence of the peculiar formation characteristics of the first stars, at the center of a minihalo whose gas cooling is dominated by the little efficient primordial chemistry; the authors suggested that this phase (called a dark star) could prevent the formation of the first stars, and be a new phase of stellar evolution. Iocco (2008) and Freese, Spolyar, and Aguirre (2008) noticed that, if a star does eventually form, the process of WIMP capture by scattering could be so efficient that their subsequent accumulation and annihilation inside the celestial object may provide an energy source comparable or even exceeding its nuclear luminosity. Motivated by these works, Iocco et al. (2008) and Freese et al. $(2008 \mathrm{~b}, \mathrm{c})$ have studied the early pre-MS phase, in which the baryonic structure is sustained by the annihilation of the DM accreted inside it by gravitational contraction, with the help of numerical codes. Both the groups find this phase is transient, although the techniques adopted are different and the details of the treatment lead to different duration estimates; they both conclude, however, that the collapse must continue at the end of this process, which for the sake of simplicity we call the Adiabatic Contraction $(A C)$ phase. In Iocco et al. (2008) (hereafter, I08), we have also studied the pre-MS phase 
of these stars in presence of annihilating scattered and captured DM ( $S C$ phase) and followed the evolution of stellar models of different mass until the end of the helium burning, in different DM environments. We find the duration of the MS is dramatically prolonged, up to a potentially everlasting phase (depending on the choice of parameters, see later): the energy released by the annihilating DM can support the core at temperatures low enough that nuclear reactions are never ignited. Yoon, Iocco, and Akiyama (2008) and Taoso et al. (2008) also studied the $S C$ phase, confirming our results with different codes and carrying their analysis further. These proceedings are based on the results obtained by I08, to which we address the reader for detailed referencing and more quantitative details: here we aim to a more qualitative description of the physical processes at the basis of this class of objects.

\section{Adiabatic Contraction phase}

Early stars are thought to form in halos of $\mathrm{M}_{h} \sim 10^{6} \mathrm{M}_{\odot}$ and virial temperature $\mathrm{T} \lesssim$ $10^{4} \mathrm{~K}$ at redshift $\mathrm{z} \sim 20$. The primordial, metal free composition of the gas, the absence of strong magnetic fields and turbulence make early star formation very different from the one in the older universe. Simulations tell us that the result of such peculiar environment are massive $\left(30-300 \mathrm{M}_{\odot}\right)$ stars that form in the very center of the halo (accurate review and referencing in the First Stars III proceedings, 2008). The collapse of the baryonic material "pulls" also the collisional DM towards the center, thus contributing to the build-up of a central "spike" of DM; if that is indeed made of self-annihilating particles (as WIMP neutralinos are), its higher concentration in the center of the halo causes a huge enhancement of the annihilation term:

$$
\frac{d L_{\mathrm{DM}}}{d V}=f c^{2} \frac{\rho^{2}\langle\sigma v\rangle}{m_{\chi}}
$$

with $\rho$ the local DM density, $m_{\chi}$ the neutralino mass - which we take to be $100 \mathrm{GeV}-$, $\langle\sigma v\rangle$ the thermally-averaged annihilation rate. It is worth noting that this energy term depends on the self-annihilation rate, whose value can be quite safely established through cosmological arguments (see e.g. the latest review on particle DM physics, Bertone et al. $2005)$ as $\langle\sigma v\rangle=3 \times 10^{-26} \mathrm{~cm}^{3} \mathrm{~s}^{-1}$. A fraction $(1-f)$ of the whole energy is emitted in the form of neutrinos, and therefore lost by the system, whereas all the other products of annihilation can quickly thermalize inside the gas already at densities $n \sim 10^{13} \mathrm{\#} / \mathrm{cm}^{3}$, as shown by Spolyar et al. (2008); we take $f \sim 2 / 3$, a typical value for a neutralino annihilation. In order to study this peculiar object we have modified the Padova Stellar Evolution code to take into account the energy released by DM annihilation. The initial models have been prepared as follows: $(i)$ the "stellar" profile (namely the baryonic structure) has been obtained by "pumping" a Zero Age Main Sequence star with an artificial energy source (as much as permitted by the stability of our numerical code); for a $100 \mathrm{M}_{\odot}$ star, the resulting structure is an object of radius $\mathrm{R}_{*}=1.2 \times 10^{14} \mathrm{~cm}$ and effective temperature $T \sim 5 \times 10^{3} \mathrm{~K}$; $(i i)$ the DM profile has been obtained in the approximation of an adiabatically contracted DM profile (from an original NFW) which has been matched to the baryonic structure in the center, which dictates the gravitational potential. For details on the characteristics of the halo and on the adiabatic contraction approximation used we address the reader to I08. This adiabatically contracted DM profile represents the initial model, at the time we start our analysis: we have implemented in our code a routine which allows to follow the adiabatic contraction of the DM inside our stellar object, account for the energy released by its annihilation, and so self-consistently describe the evolution of the DM coupled to the baryons. In this way we can follow the 

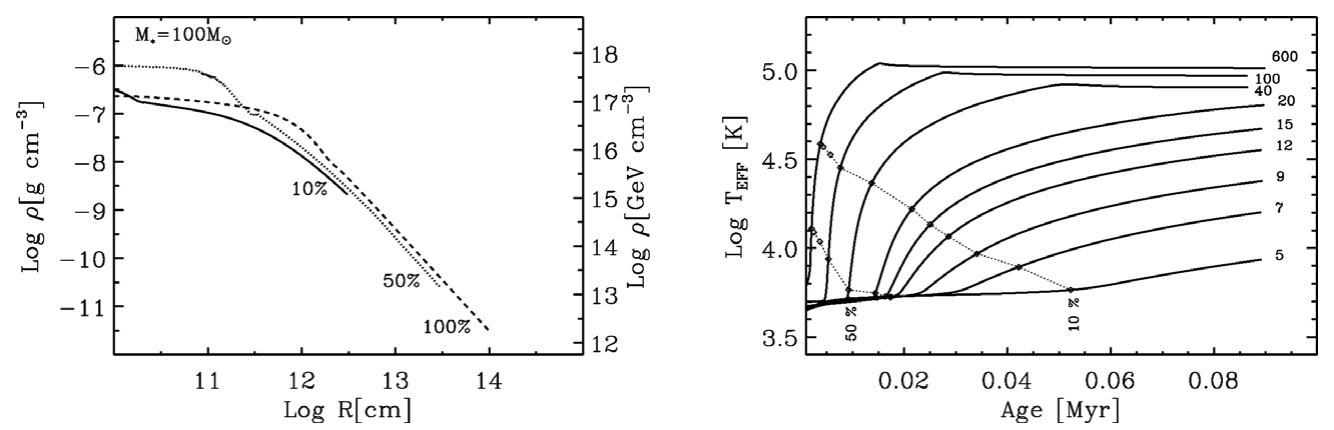

Figure 1. In the left panel: DM density profiles, truncated at the stellar radius, for a $100 \mathrm{M}_{\odot}$ at different times during the $A C$ phase. In the right panel: evolution of the effective temperature for all stellar models, benchmarked by the relative contribution of DM annihilation to the total luminosity of the star. See text for details.

contraction of the protostar toward the Main Sequence including all relevant energy sources (gravitational, DM annihilation, and possibly nuclear) and adopt a complete equation of state for the gas.

For the adopted AC parameters, DM annihilation is able to support the baryonic structure in the very early phases of gravitational contraction, in all our selected stellar models (5-600 $\left.\mathrm{M}_{\odot}\right)$ The protostars contract "pulling in" more and more DM until an equilibrium is reached in very short times, $\mathcal{O}\left(10^{2} \mathrm{yr}\right)$.

However, this phase is doomed to be transient. As the annihilation proceeds, the star contracts because of the shrink of DM luminosity caused by its consumption. But this further contraction is not able to restore the previous DM density profile, and the star finds its equilibrium at a lower luminosity. In this phase the star descends slowly along the Hayashi line. Besides consumption due to annihilation, the star continuously loses the external shells of DM, because of the different response of baryons and DM to the growing gravitational potential. Eventually the contraction of DM becomes not fast enough to restore an "efficient" cusp and the DM luminosity is no more able to balance the stellar energy losses. At this point the star keeps on contracting on a typical Kelvin-Helmholtz time-scale, terminating the AC phase and moving toward the main sequence. By defining the duration of this phase $\tau_{A C}$ as the the time needed from the DM annihilation to scale from $100 \%$ to $50 \%$ of the total luminosity of the object, we find typical values of order $\tau_{\mathrm{AC}} \sim 10^{3} \mathrm{yr}$, ranging from $\tau_{\mathrm{AC}}=2.1 \times 10^{3} \mathrm{yr}$ for a $600 M_{\odot}$ star up to $\tau_{\mathrm{AC}}=1.8 \times 10^{4} \mathrm{yr}$ for a $9 M_{\odot}$ star. The rate of gravitational energy release is larger in more massive stars, so it comes as no surprise that the stalling phase is shorter for higher mass stars. In Figure 1 we show the effective temperature evolution for different stellar models and the DM profile inside the baryonic structure for a $100 \mathrm{M}_{\odot}$ star at different times during the stellar collapse. The benchmark values correspond to the fractional contribution of DM annihilation to the total luminosity.

\section{Scattering and Capture phase}

The other physical mechanism able to concentrate DM inside a star is the capture of WIMPs by means of elastic scattering with the gas particles that constitute the object. The captured WIMPs thermalize with the gas and eventually reach a thermally relaxed 
state inside the star: the density profile $n_{\chi}$ is

$$
n_{\chi}(R)=n_{\chi}^{c} \exp \left(-R^{2} / r_{\chi}^{2}\right), \quad n_{\chi}^{c}=\frac{C_{*} \tau_{\chi}}{\pi^{3 / 2} r_{\chi}^{3}}, \quad r_{\chi}=c\left(\frac{3 k T_{c}}{2 \pi G \rho_{c} m_{\chi}}\right)^{1 / 2} ;
$$

where $T_{c}$ and $\rho_{c}$ are the stellar core temperature and density, respectively, and $C_{*}$ the WIMP capture rate, discussed in the following. It can be seen that $r_{\chi}$ is in general much smaller than the star core, $r_{\chi} \sim 10^{9} \mathrm{~cm}$ for a $100 \mathrm{M}_{\odot}$ star. For each volume element, the DM annihilation energy released is given by Equation 2.1; however, capture and annihilation reach the equilibrium on timescales much shorter than the stellar lifetime (see Iocco 2008 and I08 for a more detailed discussion of this issue), and the amount of energy released inside the star, the "dark luminosity" $L_{D M}$ reads $L_{D M}=f m_{\chi} C_{*}$, being dictated by the capture rate, $C_{*}$. The latter has the characteristic of a scattering term, and can therefore be cast as:

$$
C_{*} \propto M_{*} v_{\mathrm{esc}}^{2} \frac{\sigma_{0} \rho}{m_{\chi}} .
$$

with $\sigma_{0}$ the elastic scattering cross section between WIMP and baryons, $\rho$ the DM density outside the star, $v_{\text {esc }}$ the escape velocity at the surface of the star, and the subscript * referring to stellar quantities throughout this paper. We refer to I08 and references therein for a more quantitative discussion of the capture rate, but we wish here to stress on few peculiarities of the $S C$ process. The energy is indeed provided by means of DM annihilation in the bosom of the star; however, the bottleneck of the capture/scattering mechanism is given by capture: the star cannot burn more WIMPs than it accretes. Therefore, at the equilibrium, the annihilation is dictated by the capture and shows the parameter dependence of a scattering process. Also, this process is sensitive to the WIMPs that stream through the star, and thus the halo plays the role of a "reservoir", virtually unexhaustable $\dagger$. It depends on $\sigma_{0}$ rather than $\langle\sigma v\rangle$, and linearly on the environmental DM density $\rho$. However, when the object is in the $A C$ phase, the $S C$ mechanism is playing little role due to the very low density of the object (which makes it an inefficient "net" for capturing DM) and of the very long equilibrium timescale at these stages, much longer than the Kelvin-Helmholtz time. By contracting, the star enhances the efficiency of the $S C$ process and DM annihilation contributes to the stellar luminosity.

For $\sigma_{0}=10^{-38}$ (at the level of the current upper limit for the spin-dependent elastic scattering cross section (Desai et al. 2004), and an environment density $\rho=$ $10^{12} \mathrm{GeV} / \mathrm{cm}^{3}$ (a likely value achieved around the star, see Figure 1), the energy provided by the DM annihilation inside the star is able to support all our models before they get to the ZAMS, therefore not igniting nuclear reactions. Their locus on the HR diagram can be observed in Figure 2, compared with their ZAMS one. Lower values of $\rho$ or $\sigma_{0}$ make the DM contribution smaller, and the star can contract increasingly, thus progressively enabling the ignition of nuclear reactions. Stars in the white region of the right panel of Figure 1 do never ignite nuclear reactions; stars below the solid curve, in the shaded area do. The closer to the curve, the longer their lifetimes will be, as energy contribution required by nuclear burning is lower, and their chemical evolution is slower, see I08.

$\dagger$ The DM mass-energy content of the halo is much bigger than the energy used by the star: only approximately $10 \mathrm{M}_{\odot}=10^{-5} \mathrm{M}_{h}$ are entirely converted into energy in $10 \mathrm{Gyr}$, at a rate $10^{39} \mathrm{erg} / \mathrm{s}$. Only $0.1 \mathrm{M}_{\odot}$ of $\mathrm{DM}$ can support a $100 \mathrm{M}_{\odot}$ star for ten times longer than its natural lifetime, $10^{6} \mathrm{yr}$. 

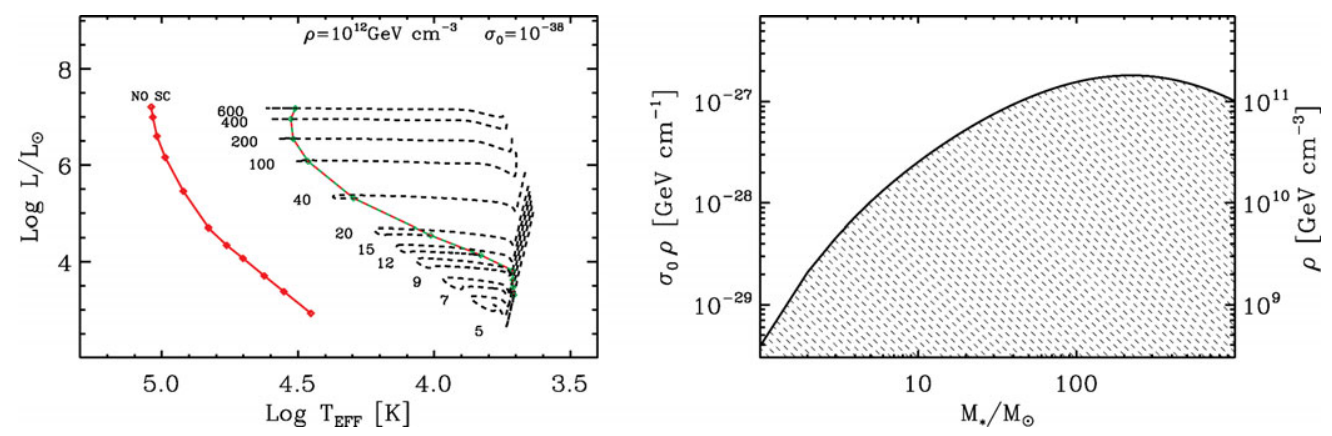

Figure 2. In the left panel: HR diagram for metal-free stars in presence of a DM environmental density $\rho=10^{12} \mathrm{GeV}$, compared to their normal position in the HR; sold curve labeled NO SC. For all masses, the ZAMS is never reached, as stars are entirely supported by DM annihilation. In the left panel: stars in the white region, above the solid line never ignite nuclear reactions, being "frozen" in their evolution. In the shaded area they do evolve at different rates, being only partially supported by DM burning. See text for details.

\section{Conclusions}

We have studied the effects of WIMP dark matter annihilation from the late stages of stellar formation through the end of helium burning. Addressing the reader to I08 for quantitative details, here we wish to stress the fundamental physical difference between the $S C$ mechanism, which needs a weak process to mediate the capture and thermalization of DM particles (and therefore the DM density profile inside the star), and the $A C$ one, where the DM density profile is dictated by gravitational contraction only. The difference in the two physical processes dictates diverse characteristics for the two phases: the $A C$ is transient, and takes place early in the pre-Main Sequence evolution of the star, when it is almost at the proto-stellar stage. The $S C$ phase becomes active when the star is at the bottom of the Hayashi track or later in the pre-MS evolution, and it can dramatically extend the stellar life, up to orders of magnitude more than its standard lifespan, depending on parameters.

\section{References}

Bertone, G., Hooper, D., \& Silk, J., 2005, Phys. Rep. 405, 279

Desai, S. et al. [Super-Kamiokande Collaboration], 2004, Phys. Rev. D, 70, 109901

"First Stars III", 2008, AIP Conf. Proc., 990, T. Abel, A. Heger, and B. W. 'O. Shea eds

Freese, K., Spolyar, D., \& Aguirre, A., arXiv:0802.1724 [astro-ph]

Freese, K., Gondolo, P., Sellwood, J. A., \& Spolyar, D., [Freese et al. (2008b)] arXiv:0805.3540 [astro-ph]

Iocco, F., 2008, ApJ, 677, L1

Iocco, F., Bressan, A., Ripamonti, E., Schneider, R., Ferrara, A., \& Marigo, P., MNRAS in press, arXiv:0805.4016 [astro-ph]

Yoon, S. C., Iocco, F., \& Akiyama, S., arXiv:0806.2662 [astro-ph]

Spolyar, D., Freese, K., \& Gondolo, P., 2008, Phys. Rev. Lett., 100, 051101

Taoso, M., Bertone, G., Meynet, G., \& Ekstrom, S., arXiv:0806.2681 [astro-ph]. 